Research Paper

\title{
Clinical insomnia and associated factors in failed back surgery syndrome: a retrospective cross-sectional study
}

\author{
Soon Young Yun, Do Heon Kim, Hae Yoon Do, and Shin Hyung Kim ${ }^{\bowtie}$ \\ Department of Anesthesiology and Pain Medicine, Anesthesia and Pain Research Institute, Yonsei University College of Medicine, Seoul, Republic of Korea \\ $\triangle$ Corresponding author: Shin Hyung Kim MD, PhD., Department of Anesthesiology and Pain Medicine, Anesthesia and Pain Research Institute, Yonsei \\ University College of Medicine, 50 Yonsei-ro, Seodaemun-gu, Seoul 120-752, Republic of Korea Tel: 82-2-2228-7500, Fax: 82-2-364-2951, E-mail: tessar@yuhs.ac \\ (C) Ivyspring International Publisher. This is an open access article distributed under the terms of the Creative Commons Attribution (CC BY-NC) license \\ (https://creativecommons.org/licenses/by-nc/4.0/). See http://ivyspring.com/terms for full terms and conditions.
}

Received: 2016.12.26; Accepted: 2017.03.15; Published: 2017.04.09

\begin{abstract}
Background Insomnia frequently occurs to patients with persistent back pain. By worsening pain, mood, and physical functioning, insomnia could lead to the negative clinical consequences of patients with failed back surgery syndrome (FBSS). This retrospective and cross-sectional study aims to identify the risk factors associated with clinical insomnia in FBSS patients.

Methods A total of 194 patients with FBSS, who met the study inclusion criteria, were included in this analysis. The Insomnia Severity Index (ISI) was utilized to ascertain the presence of clinical insomnia (ISI score $\geq 15$ ). Logistic regression analysis evaluates patient demographic factors, clinical factors including prior surgical factors, and psychological factors to identify the risk factors of clinical insomnia in FBSS patients.

Results After the persistent pain following lumbar spine surgery worsened, $63.4 \%$ of patients reported a change from mild to severe insomnia. In addition, $26.2 \%$ of patients met the criteria for clinically significant insomnia. In a multivariate logistic regression analysis, high pain intensity (odds ratio $(\mathrm{OR})=2.742,95 \%$ confidence interval $(\mathrm{Cl}): 1.022-7.353, P=0.045)$, high pain catastrophizing $(\mathrm{OR}=4.185,95 \% \mathrm{Cl}: 1.697-10.324, P=0.002)$, greater level of depression $(\mathrm{OR}=3.330,95 \% \mathrm{Cl}$ : $1.127-9.837, P=0.030$ ) were significantly associated with clinical insomnia. However, patient demographic factors and clinical factors including prior surgical factors were not significantly associated with clinical insomnia.
\end{abstract}

Conclusions Insomnia should be addressed as a critical part of pain management in FBSS patients with these risk factors, especially in patients with high pain catastrophizing.

Key words: failed back surgery syndrome; insomnia; risk factors; pain severity; depression; pain catastrophizing.

\section{Introduction}

Failed back surgery syndrome (FBSS), also called post-laminectomy syndrome, is a term that defines an unsatisfactory result of a patient who suffered from spinal surgery regardless of the type and intervention area [1]. FBSS is associated with chronic pain in the lumbosacral area with or without radiation to the leg [1]. This can give rise to persistent pain and disability which often impose serious emotional and financial burden on the patient [2].

Insomnia has long been known to be related to chronic pain conditions. Insomnia is caused by reduced sleep quality and duration, a greater amount of time of fall asleep, poor daytime function, and greater sleep dissatisfaction and distress [3]. A study has recently reported that a significantly high prevalence of sleep deprivation was found in patients with FBSS [4]. Because insomnia has been shown to worsen pain, mood, and physical functioning, it could negatively impact the clinical outcomes of patients with FBSS. Thus, identifying subgroups of the FBSS population with a high risk for insomnia has a clinical importance. Understanding these factors will help 
clinicians educate patients and will facilitate the formulation of more effective treatment plans. However, many clinics have insufficient resources or expertise that provide a detailed sleep assessment for FBSS patients who are complaining of insomnia.

The Insomnia Severity Index (ISI) is a self-report measure to assess patient's perception of insomnia and the levels of severity [5]. The index is comprised of seven items of the subjective symptoms and daytime outcomes of insomnia along with the degree of distress that those difficulties cause. The questionnaire is brief and easy to score. Also, the ISI has demonstrated useful psychometric properties: convergent validity, discriminant validity, and test-retest reliability [5].

The aim of this retrospective and cross-sectional study is to identify factors relevant to clinical insomnia based on ISI scores in FBSS patients among demographic factors, clinical factors including prior surgical factors, and psychological factors.

\section{Methods}

\section{Study population}

The institutional review board approved this retrospective and cross-sectional study. In this study, the sample population was patients with FBSS, who were treated for pain between January and December 2015 at our outpatient clinic. Data was obtained from a clinical data retrieval system in our institution. For this study, we defined FBSS as persistent or recurrent back and/or leg pain that occur notwithstanding lumbar (open) spinal surgery [1]. Patients who received their last surgery $\geq 3$ months before the start of this study were included. The persistence of pain beyond 3 months established chronicity. We did not include patients who underwent percutaneous pain interventions such as epidural neuroplasty. We excluded patients with current infectious diseases, cancer, and psychiatric and neurologic disorders that would preclude completion of pain-related questionnaires. Patients were also excluded if they had major structural pathologies of the lumbar spine including traumatic spinal cord injuries, infections, or neoplasms. In addition, patients who were reported to have been diagnosed with primary insomnia or with obstructive sleep apnea or peripheral neuropathy were excluded.

\section{Insomnia severity measurement}

The severity and prevalence of insomnia were assessed by using the ISI data which was recorded at the first visit. The ISI includes seven items that assess severity of sleep onset, sleep-maintenance difficulties, patient satisfaction with current sleep pattern, insomnia interference with daily functioning, noticeable impairment of abilities attributed to sleep deprivation, and the degree of distress caused by sleep problem. Each item is graded on a five-point scale (0 to 4$)$, so that the global score range from 0 to 28, with higher scores indicating more severe insomnia. According to the recommended score interpretation guidelines [5], a global score of 0-7 indicates "no clinically significant insomnia," 8-14 indicates "sub-threshold insomnia," 15-21 indicates "moderate clinical insomnia," and 22-28 indicates "severe clinical insomnia." For this study, we defined clinical insomnia as an ISI global score $\geq 15$.

\section{Data measures and assessments}

Patient data on age, gender, body mass index (BMI), duration of pain, pain score measured on a 0 to 10 numeric rating scale (NRS; we asked patients to rate their worst back and/or leg pain that they felt during the last 2 weeks), current medications, presence of medical comorbidities (diagnosed hypertension, diabetes mellitus, heart diseases, or neurologic diseases currently requiring medical treatment), detailed lumbar spinal surgical history (number of prior surgery and type of surgery), presence of pain-related compensation (workers' compensation patients and those involved in accidents with insurance coverage/involvement), presence of symptoms suggesting neuropathic pain (radiating pain and/or symptoms including dysesthesia or allodynia, burning or coldness, "pins and needles" sensation, numbness and itching), and presence of comorbid musculoskeletal pain (musculoskeletal pain in areas other than the back and legs, such as the neck, shoulder, or joints), and level of anxiety or depression as assessed by the 14 items on the Hospital Anxiety and Depression Scale (HADS) [6]. The 14 HADS items, each scored on a 0 to 3 scale, were used to measure degree of anxiety (seven items) and depression (seven items). Thus, the two subscales range from 0 to 21 , with higher scores indicating a greater likelihood of an anxiety or depressive disorder. The cut-off value for identification of suspected cases is generally considered to be 8 [6]. The severity of pain catastrophizing was evaluated by using the Pain Catastrophizing Scale (PCS). The PCS is comprised of 13 items measuring catastrophizing thoughts or feelings accompanying the experience of pain. We ask respondents to reflect their past painful experiences and to indicate to what degree of 13 thoughts or feelings they were experienced when in pain. Each item is graded on a five-point scale $(0=$ not at all to $4=$ all the time), so the global score ranges from 0 to 52 , with higher scores indicating a greater pain catastrophizing state [7]. The cut-off value for identification of high risk cases is generally 
considered to be 30 [7]. The demographic data and pain-related clinical data, ISI, HADS, and PCS data were obtained from all patients by independent resident doctors during the preliminary medical examination.

\section{Statistical Analysis}

Continuous variables as mean \pm SD, and categorical variables as numbers (percentage) are shown to us. Logistic regression was utilized to estimate crude odds ratios (ORs) with 95\% confidence intervals (CIs) to determine the degree of correlation between candidate effect variables and clinical insomnia (ISI score $\geq 15$ ). The analyzed variables included demographic data (age, gender, BMI), duration of pain ( $<1$ year or $\geq 1$ year), pain score (NRS $<7$ or $\geq 7$ ), presence of comorbidities, multiple prior spinal surgery, type of spinal surgery, presence of a neuropathic pain component, presence of comorbid musculoskeletal pain, severity of anxiety and depression symptoms (HADS $<8$ or $\geq 8$ ), and severity of pain catastrophizing (PCS $<30$ or $\geq 30$ ). Variables with a $P$-value $<0.05$ were considered to be statistically significant and were included in a multivariate logistic regression analysis to estimate adjusted ORs and 95\% CIs. All statistical analyses were performed using the Statistical Package for the Social Sciences, version 23.0 (SPSS Inc., Chicago, IL, USA).

\section{Results}

Data from 229 patients with prior lumbar spinal surgery history who were treated for back/ leg pain at our pain clinic were acquired from electronic medical records. After excluding cases of patients who had received surgery within 3 months before the study, patients in total were assessed for eligibility. Five patients with cancer and four patients with pre-existing psychiatric and neurologic disorders such as major depressive disorder and Alzheimer's disease were excluded. Two patients who were suspected to have had diabetic neuropathy and one patient with traumatic spinal cord injury were excluded. Four patients were excluded for primary insomnia that emerged prior to pain. Seven patients with incomplete data were also excluded. Finally, a total of 194 patients with FBSS satisfied the study inclusion criteria and were included in the analyses (Figure 1).

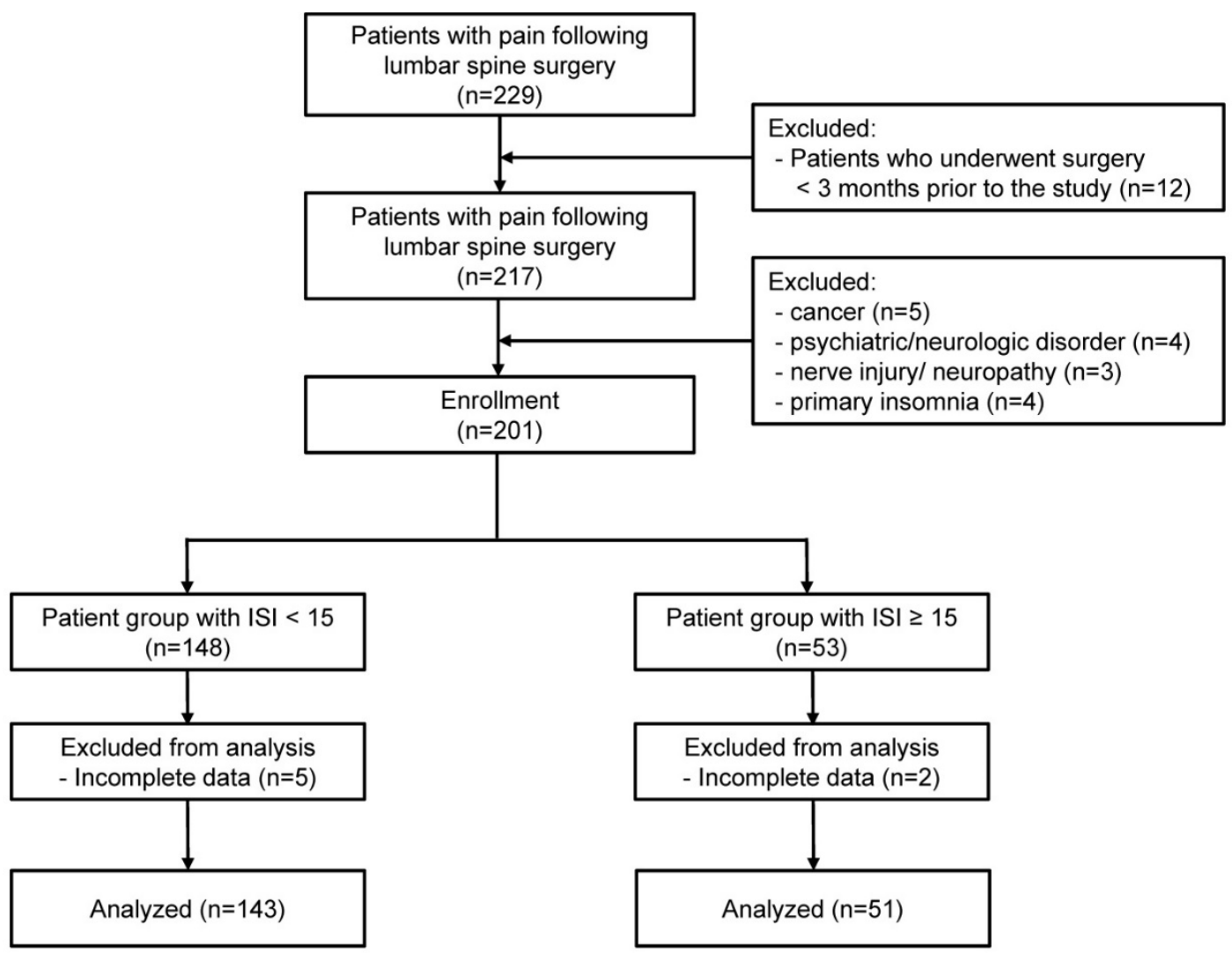

Figure 1. Flow chart of the study. ISI= Insomnia Severity Index. 
Table 1 shows patient demographics and clinical characteristics. Most patients suffered from adjacent segment diseases such as recurred disc herniation, stenosis, and instability in adjacent segments. Also, suspected causes of pain were very mixed in that $66 \%$ of patients had more than two types of suspected diagnoses. The mean global score for the ISI was 9.4, and $63.4 \%$ of patients reported from mild to severe insomnia symptoms (ISI score $\geq 8$ ) after their pain development. In $26.2 \%$ of patients, clinical insomnia with moderate to severe severity (ISI score $\geq 15$ ) was observed (Table 2).

Table 1. Demographics and clinical characteristics

\begin{tabular}{ll}
\hline & $n=194$ \\
\hline Age, years & $65.0 \pm 12.2(26-84)$ \\
Gender, M/F & $81 / 113$ \\
Body mass index, $\mathrm{kg} / \mathrm{m}^{2}$ & $24.9 \pm 3.1(16.0-34.9)$ \\
Pain duration, months & $31.9 \pm 47.3(3$ months-20 years) \\
Pain score, 0 to 10 NRS & $6.6 \pm 2.2(1-10)$ \\
Type of prior surgery, $\mathrm{n}$ & \\
Fusion & $77(39.6 \%)$ \\
Laminectomy & $89(45.8 \%)$ \\
Discectomy & $39(20.1 \%)$ \\
Other & $25(12.8 \%)$ \\
Diagnosis, $\mathrm{n}$ & \\
Recurrent disc herniation & \\
Canal/foraminal stenosis & $42(21.6 \%)$ \\
Epidural fibrosis/adhesions & $68(35.0 \%)$ \\
Spinal instability/pseudarthrosis & $16(8.2 \%)$ \\
Facet/sacroiliac joint pain & $21(10.8 \%)$ \\
Myofascial pain & $47(24.2 \%)$ \\
Surgical complications & $28(14.4 \%)$ \\
Other & $3(1.5 \%)$ \\
\hline
\end{tabular}

Values are expressed as the mean \pm SD (range) or number of patients (\%).

NRS=numeric rating scale. "Diagnosed via MRI
Table 2. Insomnia Severity Index score data

\begin{tabular}{ll}
\hline & $n=194$ \\
\hline $\begin{array}{l}\text { Global score of the Insomnia Severity Index } \\
\text { Severity of insomnia }\end{array}$ & $9.43 \pm 5.84(2-25)$ \\
0-7: no insomnia & $71(36.6 \%)$ \\
8-14: sub-threshold insomnia & $72(37.1 \%)$ \\
15-21: clinical insomnia (moderate) & $45(23.2 \%)$ \\
22-28: clinical insomnia (severe) & $6(3.1 \%)$ \\
\hline Values are expressed as the mean \pm SD (range) or number of patients (\%).
\end{tabular}

We found in univariate analysis that a high pain score (NRS $\geq 7$ ), the presence of pain-related compensation, a high level of pain catastrophizing (PCS $\geq 30$ ), and a greater level of anxiety and depression (HADS $\geq 8$ ) were all significantly related to clinical insomnia (Table 3). There were no noteworthy differences in demographic factors such as age, gender, and BMI between patients who reported clinical insomnia and those who did not. Surgery-related factors such as multiple prior surgery history and fusion surgery showed no significant association with clinical insomnia.

Multivariate logistic regression analysis showed that a high pain score (NRS $\geq 7$ ), a high pain catastrophizing level (PCS $\geq 30$ ), and a greater level of depression (HADS $\geq 8$ ) were deeply affected by clinical insomnia in our study population (Table 3). Among the above-mentioned variables, high pain catastrophizing (PCS $\geq 30$ ) was the strongest factor associated with clinical insomnia, having the highest odds ratio of 4.185 (95\% CI 1.697 - 10.324).

Table 3. Crude and adjusted odds ratios for factors associated with clinical insomnia (Insomnia Severity Index $\geq 15$ ) in 194 patients with failed back surgery syndrome: results of logistic regression analysis

\begin{tabular}{|c|c|c|c|c|c|c|}
\hline Factors & $n$ & Clinical insomnia, $n(\%)$ & Crude OR $(95 \%$ CI) & $P$-value & Adjusted OR (95\% CI) & $P$-value \\
\hline \multicolumn{7}{|l|}{ Demographic factors } \\
\hline Gender & & & & 0.923 & & 0.815 \\
\hline Male & 81 & $21(25.9)$ & 1.000 & & 1.000 & \\
\hline Female & 113 & $30(26.5)$ & $1.033(0.540-1.976)$ & & $1.109(0.466-2.637)$ & \\
\hline Age & & & & 0.162 & & 0.738 \\
\hline$<65$ years & 79 & 25 (31.6) & 1.000 & & 1.000 & \\
\hline$\geq 65$ years & 115 & $26(22.6)$ & $0.631(0.331-1.202)$ & & $0.865(0.369-2.024)$ & \\
\hline Body mass index & & & & 0.284 & & \\
\hline$<25 \mathrm{~kg} / \mathrm{m}^{2}$ & 115 & $27(23.4)$ & 1.000 & & & \\
\hline$\geq 25 \mathrm{~kg} / \mathrm{m}^{2}$ & 79 & $24(30.3)$ & $1.422(0.746-2.710)$ & & & \\
\hline \multicolumn{7}{|l|}{ Clinical factors } \\
\hline Pain duration & & & & 0.993 & & \\
\hline$<1$ year & 99 & $26(26.2)$ & 1.000 & & & \\
\hline$\geq 1$ year & 95 & $25(26.3)$ & $1.003(0.529-1.901)$ & & & \\
\hline Pain score & & & & $<0.001$ & & 0.045 \\
\hline$<7$ NRS & 77 & $8(10.3)$ & 1.000 & & 1.000 & \\
\hline$\geq 7$ NRS & 117 & $43(36.7)$ & $5.012(2.201-11.412)$ & & $2.742(1.022-7.353)$ & \\
\hline Comorbidities & & & & 0.422 & & \\
\hline No & 62 & $14(22.5)$ & 1.000 & & & \\
\hline Yes & 132 & $37(28.0)$ & $1.335(0.659-2.706)$ & & & \\
\hline Multiple prior spinal surgery & & & & 0.345 & & \\
\hline No & 150 & $37(24.6)$ & 1.000 & & & \\
\hline
\end{tabular}




\begin{tabular}{|c|c|c|c|c|c|c|}
\hline Yes & 44 & $14(31.8)$ & $1.425(0.683-2.972)$ & & & \\
\hline Fusion surgery & & & & 0.114 & & \\
\hline No & 117 & $26(22.2)$ & 1.000 & & & \\
\hline Yes & 77 & $25(32.4)$ & $1.683(0.882-3.211)$ & & & \\
\hline $\begin{array}{l}\text { Comorbid musculoskeletal pain } \\
\text { (except back and legs) }\end{array}$ & & & & 0.688 & & \\
\hline No & 160 & $43(26.8)$ & 1.000 & & & \\
\hline Yes & 34 & $8(23.5)$ & $0.837(0.352-1.991)$ & & & \\
\hline Neuropathic pain components & & & & 0.478 & & \\
\hline No & 41 & $9(21.9)$ & 1.000 & & & \\
\hline Yes & 153 & $42(27.4)$ & $1.345(0.592-3.056)$ & & & \\
\hline Compensation/legal claims & & & & $<0.001$ & & 0.121 \\
\hline No & 178 & $42(23.5)$ & 1.000 & & 1.000 & \\
\hline Yes & 16 & $9(56.2)$ & $4.163(1.462-11.856)$ & & $2.950(0.751-11.583)$ & \\
\hline \multicolumn{7}{|l|}{ Psychological factors } \\
\hline Pain catastrophizing & & & & $<0.001$ & & 0.002 \\
\hline$<30$ PCS & 144 & $19(13.1)$ & 1.000 & & 1.000 & \\
\hline$\geq 30$ PCS & 50 & $32(64.0)$ & $11.696(5.511-24.824)$ & & $4.185(1.697-10.324)$ & \\
\hline Anxiety & & & & $<0.001$ & & 0.143 \\
\hline$<8$ HADS-A & 140 & $20(14.2)$ & 1.000 & & 1.000 & \\
\hline$\geq 8$ HADS-A & 54 & $31(57.4)$ & $8.087(3.946-16.575)$ & & $2.204(0.766-6.336)$ & \\
\hline Depression & & & & $<0.001$ & & 0.030 \\
\hline$<8$ HADS-D & 143 & $20(13.9)$ & 1.000 & & 1.000 & \\
\hline$\geq 8$ HADS-D & 51 & $31(60.7)$ & $9.532(4.574-19.867)$ & & $3.330(1.127-9.837)$ & \\
\hline
\end{tabular}

\section{Discussion}

In the present study, our findings confirmed that insomnia is common in FBSS patients. We found that high pain intensity, high pain catastrophizing, and high depression were strongly associated with severe insomnia symptoms in FBSS patients. However, patient demographic factors and clinical factors including prior surgical factors were connected insignificantly with clinical insomnia in this population.

Most previous studies similarly pointed out that high pain intensity is crucially linked with insomnia in chronic pain patients [4, 8-10]. In addition to this, a bidirectional relationship between pain and sleep was also reported as relating to chronic pain [11]. Sleep disturbances appear to have increased pain through hyperalgesia and decreased ability to bear pain [12-14]. This association between sleep deprivation and persistent pain could be explained by brain structure. For instance, the periaqueductal gray region and thalamus are known to play key roles in sleep and nociception [15-18]. A recent interesting study demonstrated that spinal cord stimulation for FBSS treatment makes better sleep and this sleep improvement correlates with better pain relief [19]. As a contributor to pain, insomnia should be incorporated in treatment planning for the pain associated with FBSS.

In this study, pain catastrophizing appeared to be the strongest risk factor for clinical insomnia in FBSS patients. This result indicates that cognitive processes related to pain may play a key role in insomnia development/severity in the population. Catastrophizing is defined as an exaggerated negative mindset to endure actual or anticipated painful experiences [20]. It is a key factor in what way cognition, beliefs, coping strategies and functioning are associated with the experience of pain [21]. Catastrophizing negatively influenced the prognosis of pain and disability in patients with chronic low back pain [22]. Although few studies address how pain catastrophizing plays in the connection between insomnia and pain, an important portion of variation in clinical pain severity is attributable to pain catastrophizing, and particularly rumination about pain was mediated by sleep disturbances in a population suffering from chronic myofascial pain [23]. Patients with osteoarthritis, comorbid insomnia and a high level of catastrophizing reported increased clinical pain following increased levels of central sensitization [24]. Collectively, pain catastrophizing should be considered in the sleep-pain relationship, although the underlying cognitive processes in comorbid insomnia and chronic pain are not yet clear [25].

In this study, a greater level of depression appeared to be a risk factor for clinically important insomnia in FBSS patients. Patients with FBSS frequently suffered from poor psychological states, such as depression [26]. Patients with long-lasting pain with concurrent major depression and insomnia showed severe pain-related psychosocial impairment [27]. Also, depression and sleep disturbance are independently associated with a reduced pain threshold [28]. According to the criteria in the 
Diagnostic and Statistical Manual of Mental Disorders, insomnia is a defining symptom of major depressive disorder [27]. Thus, insomnia has traditionally been assumed to be simply a comorbid symptom of depression, and has not been recognized as a separate disorder. However, recent studies suggest that insomnia may be independent of pain and depression in chronic pain patients [27-29]. Thus, FBSS patients with depression are highly susceptible to insomnia, and insomnia may be considered a separate symptom when devising treatment strategies for this subgroup, which is more prone to poor clinical outcomes.

In the general adult population, age and gender are confirmed demographic risk factors for insomnia, with a greater prevalence in women and older adults [3]. However, in chronic pain conditions, pain-related clinical characteristics seem to be more important than patient demographics in insomnia. Patient demographic factors were not significantly associated with clinical insomnia in patients with FBSS in this study. In several studies dealing with insomnia in chronic pain as well as our study, no relationship between insomnia and age, gender, or BMI was reported $[4,8,9]$.

Before the study, we expected that surgical factors, such as multilevel fusion surgery or multiple prior surgery history, might influence sleep in the FBSS population. However, we observed a lack of significance between prior surgical history and current insomnia severity in this population. Our results showed that current pain severity and poor psychological states may contribute more to clinical insomnia than prior surgical history in this population. The presence of pain-related compensation was significantly associated with clinical insomnia in FBSS on univariate analysis. In fact, a significant number of FBSS patients are concerned in workman's compensation or personal injury lawsuits [2]. Patients in workman's compensation tend to have worse lumbar fusion consequences and lower postoperative return to work rates [30, 31]. However, there is not currently any clinical literature that discusses the sleep-pain relationship in workman's compensation patients with FBSS. In this study, the number of patients was not sufficient to unequivocally demonstrate an association between insomnia and compensation. Thus, more studies are required to evaluate the sleep-pain relationship in workman's compensation patients with FBSS.

Our study has some limitations. First, the study was managed in a single clinical setting that includes a selected study population with a homogeneous racial background. Thus, one must be cautious when generalizing these results to other populations. Second, use of a cross-sectional study limits the ability to make causal inferences between insomnia and current pain or sleep treatments. Also, the ISI does not include detailed parameters on the frequency or duration of insomnia and medication use, which could influence the severity of insomnia. Finally, this study was based on the subjective assessment of insomnia. Although the ISI has obvious advantages over other available measurements of insomnia in busy clinical settings, it seems important to include objective assessments of sleep with polysomnographic or actigraphic evidence [32].

In conclusion, our results demonstrated that pain intensity, pain catastrophizing, and depression were significantly associated with clinical insomnia in FBSS patients. Insomnia should be addressed as a critical part of pain management in FBSS patients with these risk factors, especially in patients with high pain catastrophizing.

\section{Study Approval}

This study was approved by the Institutional Review Board of Severance Hospital, Yonsei University Health System (ref: 4-2016-0598).

\section{Competing Interests}

The authors have declared that no competing interest exists.

\section{References}

1. Bordoni B, Marelli F. Failed back surgery syndrome: review and new hypotheses. J Pain Res. 2016;9:17-22.

2. Onesti ST. Failed back syndrome. Neurologist. 2004;10:259-64.

3. Roth T. Insomnia: definition, prevalence, etiology, and consequences. J Clin Sleep Med. 2007;3:S7-10.

4. Artner J, Cakir B, Spiekermann JA, et al. Prevalence of sleep deprivation in patients with chronic neck and back pain: a retrospective evaluation of 1016 patients. J Pain Res. 2013;6:1-6.

5. Bastien $\mathrm{CH}$, Vallières $\mathrm{A}$, Morin $\mathrm{CM}$. Validation of the Insomnia Severity Index as an outcome measure for insomnia research. Sleep Med. 2001;2:297-307.

6. Bjelland I, Dahl AA, Haug TT, et al. The validity of the Hospital Anxiety and Depression Scale: an updated literature review. J Psychosom Res. 2002;52:69-77.

7. Sullivan MJ, Bishop SR, Pivik J. The pain catastrophizing scale: development and validation. Psychol Assess. 1995;7:524

8. Visscher CM, Lobbezoo F, De Boer W, et al. Psychological distress in chronic craniomandibular and cervical spinal pain patients. Eur J Oral Sci. 2001;109:165-71.

9. Marin R, Cyhan T, Miklos W. Sleep disturbance in patients with chronic low back pain. Am J Phys Med Rehabil. 2006;85:430-5.

10. Kim SH, Sun JM, Yoon KB, et al. Risk factors associated with clinical insomnia in chronic low back pain: a retrospective analysis in a university hospital in Korea. Korean J Pain. 2015;28:137-43.

11. O'Brien EM, Waxenberg LB, Atchison JW, et al. Intraindividual variability in daily sleep and pain ratings among chronic pain patients: bidirectional association and the role of negative mood. Clin J Pain. 2011;27:425-33.

12. Roehrs T, Hyde M, Blaisdell B, et al. Sleep loss and REM sleep loss are hyperalgesic. Sleep. 2006;29:145-51.

13. Sutton BC, Opp MR. Sleep fragmentation exacerbates mechanical hypersensitivity and alters subsequent sleep-wake behavior in a mouse model of musculoskeletal sensitization. Sleep. 2014;37:515-24.

14. Onen SH, Alloui A, Gross A, et al. The effects of total sleep deprivation, selective sleep interruption and sleep recovery on pain tolerance thresholds in healthy subjects. J Sleep Res. 2001;10:35-42.

15. Sastre JP, Buda C, Kitahama K, et al. Importance of the ventrolateral region of the periaqueductal gray and adjacent tegmentum in the control of paradoxical 
sleep as studied by muscimol microinjections in the cat. Neuroscience. 1996;74:415-26.

16. Demarco GJ, Baghdoyan HA, Lydic R. Differential cholinergic activation of G proteins in rat and mouse brainstem: Relevance for sleep and nociception. J Comp Neurol. 2003;457:175-84.

17. Klamt JG, Prado WA. Antinociception and behavioral changes induced by carbachol microinjected into identified sites of the rat brain. Brain Res. 1991;549:9-18.

18. Casey KL, Morrow TJ, Lorenz J, et al. Temporal and spatial dynamics of human forebrain activity during heat pain: analysis by positron emission tomography. J Neurophysiol. 2001;85:951-9.

19. Ramineni T, Prusik J, Patel S, et al. The impact of spinal cord stimulation on sleep patterns. Neuromodulation. 2016;19:477-81.

20. Quartana PJ, Campbell CM, Edwards RR. Pain catastrophizing: a critical review. Expert Rev Neurother. 2009;9:745-58.

21. Sturgeon JA, Zautra AJ. Psychological resilience, pain catastrophizing, and positive emotions: perspectives on comprehensive modeling of individual pain adaptation. Curr Pain Headache Rep. 2013;17:317.

22. Picavet HS, Vlaeyen JW, Schouten JS. Pain catastrophizing and kinesiophobia: predictors of chronic low back pain. Am J Epidemiol. 2002;156:1028-34.

23. Buenaver LF, Quartana PJ, Grace EG, et al. Evidence for indirect effects of pain catastrophizing on clinical pain among myofascial temporomandibular disorder participants: the mediating role of sleep disturbance. Pain. 2012;153:1159-66.

24. Campbell CM, Buenaver LF, Finan P, et al. Sleep, pain catastrophizing, and central sensitization in knee osteoarthritis patients with and without insomnia. Arthritis Care Res. 2015;67:1387-96.

25. Byers HD, Lichstein KL, Thorn BE. Cognitive processes in comorbid poor sleep and chronic pain. J Behav Med. 2016;39:233-40.

26. Arts MP, Kols NI, Onderwater SM, et al. Clinical outcome of instrumented fusion for the treatment of failed back surgery syndrome: a case series of 100 patients. Acta Neurochir (Wien). 2012;154:1213-7.

27. Wilson KG, Eriksson MY, Joyce L, et al. Major depression and insomnia in chronic pain. Clin J Pain. 2002;18:77-83.

28. Chiu Y, Silman A, Macfarlane G, et al. Poor sleep and depression are independently associated with a reduced pain threshold. Results of a population based study. Pain. 2005;115:316-21.

29. Asih S, Neblett R, Mayer TG, et al. Insomnia in a chronic musculoskeletal pain with disability population is independent of pain and depression. Spine J. 2014;14:2000-7.

30. Taylor VM, Deyo RA, Ciol M, et al. Patient-oriented outcomes from low back surgery: a community-based study. Spine (Phila Pa 1976). 2000;25:2445-52.

31. Klekamp J, McCarty E, Spengler DM. Results of elective lumbar discectomy for patients involved in the workers' compensation system. J Spinal Disord. 1998;11:277-82.

32. O'Donoghue GM, Fox N, Heneghan C, et al. Objective and subjective assessment of sleep in chronic low back pain patients compared with healthy age and gender matched controls: a pilot study. BMC Musculoskelet Disord. 2009;10:122. 